American Journal of Pharmacology and Toxicology 5 (3): 125-132, 2010

ISSN 1557-4962

(C) 2010 Science Publications

\title{
Methamphetamine Regulation of Sulfotransferases in Rat Liver and Brain
}

\author{
${ }^{1,3}$ Tianyan Zhou, ${ }^{2}$ Chaoqun Huang, ${ }^{2}$ Yue Chen, ${ }^{2}$ Preeti Shanbhag and ${ }^{2}$ Guangping Chen \\ ${ }^{1}$ State Key Laboratory of Natural and Biomimetic Drugs, \\ Department of Pharmaceutics, Peking University, Beijing, China \\ ${ }^{2}$ Department of Physiological Sciences, Oklahoma State University, Stillwater, Oklahoma \\ ${ }^{3}$ Department of Pharmaceutics, School of Pharmaceutical Sciences, \\ Peking University Medical Health Center, Beijing, China
}

\begin{abstract}
Problem statement: Sulfotransferases (SULTs) are important phase II drug metabolizing enzymes. They also regulate the biological activities of various hormones. To the best of our knowledge, psychostimulants regulation of SULTs is basically not studied except one article reported that Methamphetamine (METH) treatment induced rat amygdale rSULT1A1 mRNA 4.3 fold by using microarray method to identify a series of candidate genes after single-dose METH treatment. The psychostimulant METH is used to treat disorders such as attention deficit, narcolepsy and obesity. It is also a highly addictive drug that causes serious social problems. The abuse of this drug leads to the damage of monoaminergic systems in the mammalian brain. It is important to understand how SULT expressions are regulated by psychostimulants. Approach: This study was performed to evaluate the effect of METH on SULT gene expressions in rat liver and brain. METH $\left(0,1,5,20 \mathrm{mg} \mathrm{kg}^{-1}\right.$ day $\left.^{-1}\right)$ was used to treat male and female rats for 7 days by oral administration. Rat livers and brains were collected $24 \mathrm{~h}$ after the final drug treatment. Western blot and real-time RT-PCR were used to investigate the effect of METH on SULT protein and mRNA expression, respectively, in rat liver and brain. Results: Proteins and mRNAs of rSULT1A1, rSULT2A1 and rSULT1E1 were induced in liver and brain of both male and female rats following METH treatment. rSULT1E1 was induced at much higher level compared to that of rSULT1A1 and rSULT2A1. Brain inductions were found to be much higher than those found in liver. Conclusion: These data suggest an important role of the central dopaminergic system in the regulation of liver and brain rSULT expressions.
\end{abstract}

Key words: Sulfotransferase, methamphetamine, psychostimulant, induction, gene regulation

\section{INTRODUCTION}

Sulfotransferases (SULTs) are one of the major superfamily of phase II drug metabolizing enzymes (Bojarova and Williams, 2008; Chapman et al., 2004; Gamage et al., 2006; Hempel et al., 2007; Kauffman, 2004; Rath et al., 2004; Runge-Morris and Kocarek, 2005; Wang and James, 2006). They catalyze the sulfation of hydroxyl-containing compounds. The substrates specificities of some SULT are very broad. Most hydroxyl-containing compounds (phenols and alcohols) are substrates for SULT isoforms. The cosubstrate for sulfation of all SULTs is adenosine 3'Phosphate 5'-Phosphosulfate (PAPS). Sulfation is widely observed in various biological processed. Various bio-signaling molecules including hormones such as hydroxysteroid and glucocorticoid, neurotransmitters such as monoamine, peptides and so on can be sulfated to alter their biological activities. Sulfation usually leads to the inactivation of biological signaling molecules, as the sulfated forms are usually unable to bind to receptors. SULTs also catalyze the sulfation of a wide range of xenobiotics. Sulfation of xenobiotics is mainly associated with detoxification: biotransformation of a relatively hydrophobic xenobiotic into a more water-soluble sulfuric ester that is readily excreted. However, there are numerous important exceptions wherein the formation of chemically reactive sulfuric esters is an essential step in the metabolic pathways leading to carcinogenic responses (Hempel et al., 2007; Gallucci and Mickle, 2006).

Methamphetamine (METH) is one of the wellknown psychostimulants. The abuse of this highly addictive drug leads to the long-lasting changes in dopaminergic pathway of Central Nervous System (CNS). METH administration has been shown to

Corresponding Author: Guangping Chen, Department of Physiological Sciences, Oklahoma State University, Stillwater, Oklahoma, 74078 Tel: (405) 744-2349 Fax: (405) 744-8263 
produce long-term decreases in numerous measures of both dopaminergic and serotonergic function such as enzyme activity, monoamine content and monoamine transmitters in experimental animals as well as in human (Quinton and Yamamoto, 2006; Gold et al., 2009; Krasnova and Cadet, 2009; Guilarte et al., 2003; Kish, 2008; Kita et al., 2003; Cadet et al., 2007; Volz et al., 2007; Fleckenstein et al., 2007; Sofuoglu and Sewell, 2009). METH toxicity is frequently reported as a potential model of drug induced Parkinsonism, which is one of the most common movement disorders of the elderly. Chronic or intermittent METH abuse may create temporary or permanent disturbances in the dopaminergic systems of the brain that may predispose individuals to Parkinsonism (Kita et al., 2003; Fisher et al., 2005; Yanai et al., 2005; Tolwani et al., 1999).

SULTs induction by hormones and other endogenous molecules has been relatively well known (Liu and Klaassen, 1996; Duanmu et al., 2001; Dunn and Klaassen, 2000; Klaassen et al., 1998; RungeMorris, 1998; Runge-Morris et al., 1999; Wu et al., 2001; Runge-Morris and Kocarek, 2005). Xenobiotic induction of SULTs is not well studied. Recent data suggest that SULTs can be induced by xenobiotics, although the mechanisms for xenobiotic induction are less known (Runge-Morris and Kocarek, 2005; 1998; Chen et al., 2008; Maiti and Chen, 2003a; 2003b). Psychostimulants regulation of SULTs is basically not studied. To the best of our knowledge, there is only one report on METH regulation of SULTs, which used microarray method to screen a series of candidate genes after single-dose METH treatment of rats (Niculescu et al., 2000). This report suggested that METH treatment induced rat brain SULT1A1 mRNA 4.3 fold in the amygdale.

Similar to Cytochrome P450 (CYP) enzymes, most SULTs are regulated by hormones that remain under control of the Central Nervous System (CNS). METH treatment is known to cause the change of dopamine in CNS. Dopamine is one of the most important endogenous neurotransmitters as well as an important endogenous substrate of SULTs (Yasuda et al., 2009; Salman et al., 2009; Lu et al., 2005). It has been demonstrated that the expression of CYPs can be regulated by dopamine receptor-linked signaling pathway by changing the hormone $(\mathrm{GH}$, thyroid hormones, glucocorticoids and so on) levels in vivo (Wojcikowski, 2004; Wojcikowski et al., 2007; 2008; Konstandi et al., 2008). CNS psychostimulants regulation of the expression of SULTs is not studied. We hypothesize that the effect of METH on monoaminergic systems in the brain may indirectly change the expression of SULTs mediated by the dopamine-related signaling pathway. In this study, we investigated the regulation of rSULT1A1, rSULT2A1 and rSULT1E1 by METH in both rat liver and rat brain.

\section{MATERIALS AND METHODS}

Materials: METH was purchased from Sigma-Aldrich (St. Louis, MO). SDS-polyacrylamide gel electrophoresis reagents were obtained from Bio-Rad (Hercules, CA). Western blot chemiluminescence reagent kits (Super Signal West Pico Stable Peroxide and Super Signal West Pico Luminol/Enhancer solutions) were purchased from Pierce Chemical (Rockford, IL). PVDF membranes used for Western blotting analyses were purchased from Millipore Corporation (Bedford, MA). TRI REAGENT for total RNA extraction was purchased from MRC (Cincinnati, $\mathrm{OH})$. M-MLV Reverse Transcriptase was obtained from Promega (Madison, WI). qPCR MasrerMix Plus with SYBR® Green I dNTP was purchased from Eurogentec (San Diego, CA). Antibodies against AST-IV (rSULT1A1) and STa (rSULT2A1) were provided by Dr. Michael W. Duffel (Division of Medicinal and Natural Products Chemistry, College of Pharmacy, The University of Iowa, Iowa city, IA). An antibody against rSULT1E1 was purchased from Biovision Inc (Boston, MA). Protein assay reagent was purchased from BioRad. All other reagents and chemicals were of the highest analytical grade available.

Animals and drug treatment: Male and Female Sprague-Dawley rats (Harlan, Indianapolis, IN), 1011 weeks old and 200-300 g body weight were used in this investigation. Rats were housed in a temperatureand humidity-controlled room and supplied with rodent chow and water for at least 1 week before use. Rats of both sexes were divided into 4 groups with four in each. METH was dissolved in sterile saline and administrated by gavage at 1,5 and $20 \mathrm{mg} \mathrm{kg}^{-1}$ day $^{-1}$ for 7 days. The corresponding group of control rats of either gender received only sterile saline. The rats were sacrificed $24 \mathrm{~h}$ after the final drug treatment. Livers and whole brains were collected, washed with sterile, ice-cold $\mathrm{NaCl}(0.9 \%$, w/v) solution and snap-frozen. Samples were stored at $80^{\circ} \mathrm{C}$ until use.

Cytosol preparation: Both liver and brain tissues were homogenized in $50 \mathrm{mM}$ Tris buffer containing $0.25 \mathrm{M}$ sucrose, $3 \mathrm{mM} \beta$-mercaptoethanol and $0.02 \%(\mathrm{v} / \mathrm{v})$ Tween-20, $\mathrm{pH}$ 7.4. All homogenates were centrifuged at $100,000 \mathrm{~g}$ for $1 \mathrm{~h}$ at $4^{\circ} \mathrm{C}$. Cytosol aliquots were collected and preserved at $-80^{\circ} \mathrm{C}$ for Western blot. 
Western blot analysis: Cytosol protein from liver (10 $\mu \mathrm{g})$ and brain $(50 \mu \mathrm{g})$ was used in a $12 \%$ polyacrylamide gel in an electrophoresis system (Novex, San Diego, CA). After running at $200 \mathrm{~V}$, the protein bands were transferred overnight at $35 \mathrm{~V}$ onto a PVDF membrane. Membranes were blocked for $1 \mathrm{~h}$ by $5 \%(\mathrm{w} / \mathrm{v})$ nonfat dry milk in Tris-Buffered Saline (TBS). For both liver and brain cytosol proteins, membranes were incubated with either rabbit anti-rat AST-IV (rSULT1A1), or rabbit anti-rat STa (rSULT2A1) or mouse anti-rat SULT1E1 (1:1000) overnight in TBST (50 mM Tris, $\mathrm{pH} 7.5,150 \mathrm{mM} \mathrm{NaCl}$ and $0.05 \%(\mathrm{v} / \mathrm{v})$ Tween 20) containing 5\% (w/v) nonfat dry milk on a shaker at $4^{\circ} \mathrm{C}$ After incubation, all membranes were washed with TBST for $3 \times 10 \mathrm{~min}$ and incubated in secondary antibody (Horseradish peroxidase-conjugated Immuno-Pure goat anti-rabbit IgG $(\mathrm{H}+\mathrm{L})$ for rSULT1A1 and rSULT2A1, Horseradish peroxidase-conjugated Immuno-Pure goat anti-mouse $\operatorname{IgG}(\mathrm{H}+\mathrm{L})$ for rSULT1E1) at $1: 8000$ dilutions in the same buffer for at least $1 \mathrm{~h}$. The membranes were washed with TBST for $3 \times 10 \mathrm{~min}$. Fluorescent band were developed with $3 \mathrm{~mL}$ of substrate containing the same volume of each Super Signal West Pico Luminol Enhancer solution and Super Signal West Pico Stable Peroxidase solution at room temperature for $5 \mathrm{~min}$. The fluorescence image was obtained using VersaDoc IMAGING SYSTEM 5000MP (BIO-RAD, Hercules, CA). The densitometric quantification of protein bands was obtained using Quantity One 4.6.5 software of VersaDoc imaging system.

Quantitative real-time PCR: Total RNA was extracted from liver using TRI REAGENT from MRC according to supplier's guidelines. The concentration and purity of the extracted RNA were checked spectrophotometrically by measuring 260/280 absorption ratios. M-MLV Reverse Transcriptase (Promega) with $1 \mu \mathrm{g}$ of total RNA was used to synthesize cDNA and $1 \mu \mathrm{L}$ of reverse-transcribed product served as the template in polymerase chain reactions. Real-time PCR was performed using the qPCR MasrerMix Plus with SYBR ${ }^{\circledR}$ Green I kit (EUROGENTEC) following the manufacturer's instruction. Primers were designed with Primer Express as follow: rActin-F320:5'AGGCCCCTCTGAACCCTAAG-3', rActin-R435: 5'AGAGGCATACAGGGACAACACA-3', GI NM_031144; $\quad$ rSULT1A1-F530: 5'AGCTGAGACACACTCACCCTGTT-3', rSULT1A1R651: 5'-ATCCACAGTCTCCTCGGGTAGA-3', GI. L19998; $\quad$ rSULT2A1-F496: 5'ATCCGTGCCTGGCTGTCTAT-3'， rSULT2A1-R642:
5'-GAGGACCAAATCCAGCTCATCT-3'. M33329; rSULT1E1- F432:

5'-GGT GGC TGG TCA TCC AAA TC-3', rSULT1E1R557:

5'-CGT GGA CTC TTT CCC TTT TCC-3', GI BC088157

Real-time PCR was performed on an 7500 Fast Real-Time PCR System. (Applied Biosystems, Foster City, CA). Initially, regular PCR products were purified with GENECLEAN Turbo (Qbiogene, Carlsbad, CA) for constructing standard curves $\left(10-10^{8}\right.$ copies). A standard curve was plotted with the Threshold Cycle (CT) Vs the logarithmic value of the gene copy number. The gene copy number of unknown samples was generated directly from the standard curve by the software Sequence Detector 1.7. At least two duplications were run for each standard or unknown sample. All gene copy numbers were normalized to rat $\beta$-Actin mRNA.

Data analysis: One-way ANOVA followed by the Dunnett's test was used to calculate the statistical significance of the difference between the control group means and methamphetamine treatment group means. In all cases, *: p<0.05 was considered significant; **: $\mathrm{p}<0.01$ was considered very significant. Data presented in the figures are means Standard Deviation $( \pm \mathrm{SD})$ of the data collected separately from at least three individual animals.

\section{RESULTS}

METH regulation of rSULT1A1, rSULT2A1 and rSULT1E1 in rat liver: In female liver, Western blot data showed that rat SULT1A1 (rSULT1A1, AST-IV) protein expression increased by about 44, 35 and $47 \%$, respectively, after $1,5,20 \mathrm{mg} \mathrm{kg}{ }^{-1}$ day $^{-1}$ METH treatment for 7 days (Fig. 1A). Those increases were statistically significant. Rat SULT 2A1 (rSULT2A1, $\mathrm{STa}$ ) in female rat liver increased $27 \%$ in protein level with the middle dose treatment of METH (Fig. 1A). METH induced female rat SULT1E1 (rSULT1E1, EST) protein level in liver 2.4-, 4.7- and 3.5-fold, respectively, in response to the three dose treatment (Fig. 1A). In male liver, rSULT1A1 protein expression was induced by about 77, 66 and 32\% respectively at low, middle and high doses treatment of METH (Fig. 1B). In male rat liver, rSULT2A1 protein expression was not significantly changed, although rSULT2A1 was decrease $40 \%$ at the high dose treatment, this was not statistically significant. In male rat liver, rSULT1E1 protein expression was significantly induced at the low and medium dosed, while it was significantly suppressed at the high dose. 


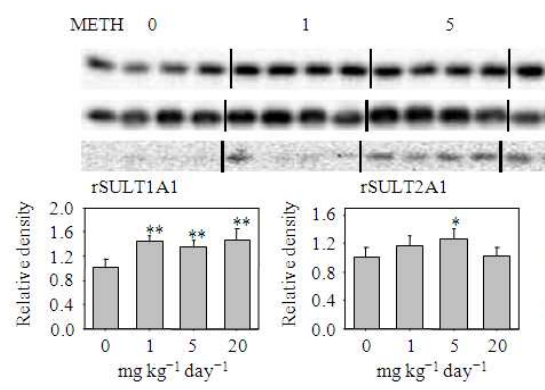

(A)

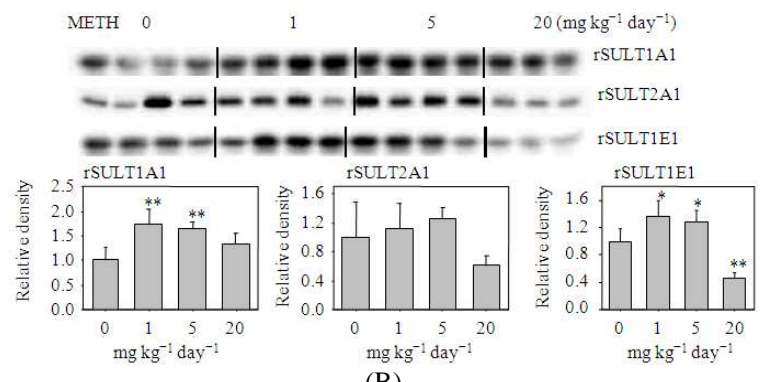

(B)

Fig. 1: Representative Western blot and densitometric analysis of rSULT1A1, rSULT2A1 rSULT1E1 in liver of (A) female and (B) male rats treated 1 week with varying doses of METH. Values were divided by the smallest densitometric value of the blot. The division factors are plotted and expressed as relative densities. *: $\mathrm{p}<0.05$; $* *$ : $<<0.01$ and $* * *: p<0.001$

Real-time PCR data demonstrated that the effect of METH treatment on the mRNA expressions of the three SULT isoforms (Fig. 2) was in good agreement with that of protein expressions (Fig. 1). This applies to all three doses treatment to all the three isoforms of SULT. This suggests that the regulation of METH on SULT expression is on the gene transcription level.

METH regulation of rSULT1A1, rSULT2A1 and rSULT1E1 in rat brain: The Western blot results and the densitometric analysis (Fig. 3A) suggested that rSULT1A1, rSULT2A1 and rSULT1E1 protein in female rat brain is significantly induced by the treatment of METH in the dose range between 1-20 mg $\mathrm{kg}^{-1}$ day $^{-1}$. The corresponding protein expression of rSULT1A1 increased by about $70 \%, 80 \%$ and $70 \%$; of rSULT2A1 increased by $113 \%, 131 \%$ and $90 \%$ and of rSULT1E1 increased by about 4.7-, 2.6-and 1.2-fold respectively, after $1,5,20 \mathrm{mg} \mathrm{kg}{ }^{-1}$ day $^{-1}$ METH treatment (Fig. 3A). The induction of female rat brain rSULTs was much greater than that found in female rat liver.
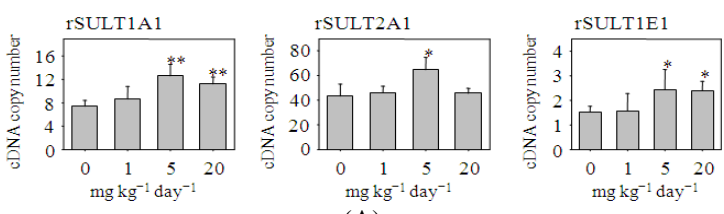

(A)
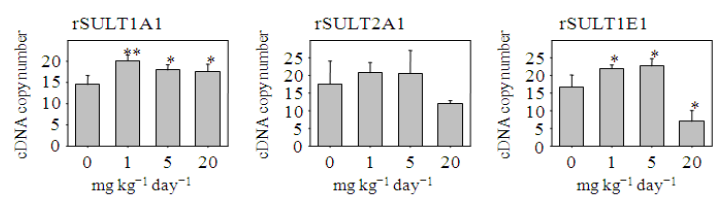

(B)

Fig. 2: rSULT1A1, rSULT2A1 and rSULT1E1 mRNA expression in livers of (A) female and (B) male rats treated 1 week with varying doses of METH. Rat $\beta$-actin was used as control for realtime PCR. Relative copy number of rSULT1A1, rSULT2A1 and rSULT1E1 mRNA were standardized by using rat $\beta$-actin mRNA. Induction fold was calculated by dividing the copy number of rSULT mRNA in METHtreated rats by the copy number of corresponding rSULT mRNA in control rats. $*: \mathrm{p}<0.05$ and $* *: \mathrm{p}<0.01$

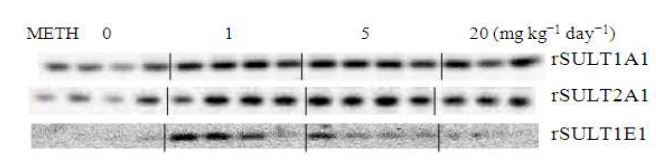

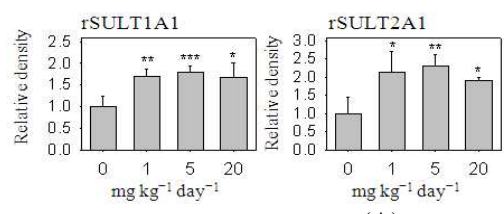

(A)

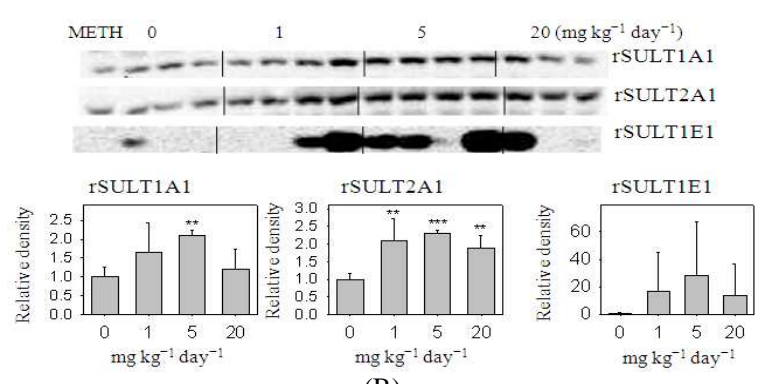

(B)

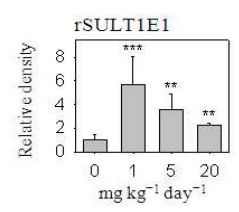
20 ( $\left.\mathrm{mg} \mathrm{kg}^{-1} \mathrm{day}^{-1}\right)$
Fig. 3: Representative Western blot and densitometric analysis of rSULT1A1, rSULT2A1 and rSULT1E1 in the brain of (A) female and (B) male rats treated 1 week with varying doses of METH. Values were divided by the smallest densitometric value of the blot. The division factors are plotted and expressed as relative densities. *: $\mathrm{p}<0.05 ; * *$ : $\mathrm{p}<0.01$ and $* * *: \mathrm{p}<0.001$ 
In male rat brain, rSULT1A1 protein level increased by about 66,110 and $19 \%$, respectively, in response to the treatment of $1,5,20 \mathrm{mg} \mathrm{kg}^{-1} \mathrm{day}^{-1}$. The induction of rSULT1A1 was not statistically significant in low and high doses treatment. rSULT2A1 protein expression was found dramatically induced in male rat brain (Fig. 3B). rSULT2A1 protein level increased by about 109,132 and $88 \%$, respectively, in response to the three dose treatment. rSULT1E1 protein level in male rat brain was induced at a very high level, increased up to about 16-, 27-, 13-fold, respectively, after $1,5,20 \mathrm{mg} \mathrm{kg}^{-1}$ day $^{-1}$ METH treatment. The induction of male rat brain SULT1E1 was not statistically significant due to huge variation between individuals. The induction of male rat brain rSULT1A1, rSULT2A1 and rSULT1E1 was much greater than that found in male rat liver.

\section{DISCUSSION}

SULTs catalyze the sulfation of xenobiotics and different biosignaling molecules including hydroxysteroid hormones, thyroid hormones, glucocorticoid hormones, neurotransmitters, bile acids, heparan and so on. Some isoforms of SULTs, e.g., SULT1A1, have very broad substrate specificity and play important roles in xenobiotics detoxification. Other SULTs, e.g., SULT1E1, have high specificity for only endogenous substrates. Although some SULT isoforms have been isolated and characterized, the biological functions of SULTs remain largely unknown. Studies on cytosolic SULTs have been mainly focused on drug metabolism and detoxification. We believe that SULTs are not only important for xenobiotic detoxification but also play important biological roles in the regulation of the activities of various biosignaling molecules and their functions. The malfunction of SULTs will therefore lead to diseases.

METH is a psychostimulant used to treat several disorders, including attention deficit, narcolepsy and obesity (Cadet et al., 2007; Fleckenstein et al., 2007). Over use of this drug causes drug dependence and addiction and cause serious social problems. We are interested to investigate the roles that SULTs play under physiological and various pathological conditions and to understand the importance of SULTs for general human health. METH is known to cause hormone level changes in vivo, we are interested to understand how METH regulate SULTs in the drug detoxifying tissue rat liver and in the center of the nervous system rat brain. SULT1A1 is the major phenol-sulfating SULT and has very broad substrate specificity. It not only sulfates simple xenobiotic phenols with high activity but also sulfates many endogenous phenolic compounds, including various hormones and neurotransmitters. SULT1A1 is also widely distributed in different tissues. SULT2A1 mainly catalyzes the sulfation of alcohol compounds; it is also the major enzyme to catalyze the sulfation of hydroxysteroids, especially for DHEA. SULT1E1 is specific for the sulfation of estrogens; it is also the major enzyme to catalyze the sulfation of thyroid hormones (Wu et al., 2008; Santini et al., 1993). In this study, these three isoforms were selected for the investigation of METH effect in rat liver and brain.

Our experimental results suggested that the simple phenol SULT, rSULT1A1 (it also catalyzes the sulfation of neurotransmitters) is induced by METH treatment in both rat liver and brain. The hydroxysteroid SULT, rSULT2A1, is somewhat less inducible by METH. The estrogen and thyroid SULT, rSULT1E1, is very sensitive to the treatment of METH. In general, the effect of METH on rat brain SULTs is much higher than that of rat liver.

Genes encoding different SULT isoforms expression can be regulated through endogenous hormones (growth hormone, sex hormones, thyroid hormones, glucocorticoids) and immune system (cytokines) in different ways and at various levels, being also tissue-dependent (Liu and Klaassen, 1996; Duanmu et al., 2001; Dunn and Klaassen, 2000; Klaassen et al., 1998; Runge-Morris, 1998; RungeMorris et al., 1999; Wu et al., 2001; Runge-Morris and Kocarek, 2005; Chen et al., 2008; Maiti and Chen, $2003 a ; 2003 b)$. Glucocorticoid-inducible rat hepatic SULT1A1 gene transcription occurs through a Glucocorticoid Receptor (GR)-mediated mechanism, a low dose of a synthetic glucocorticoid, can have physiologically significant effects attributable to the increased expression of SULT1A1 (Runge-Morris and Kocarek, 2005). Glucocorticoids have been known as important regulatory factors in the control of rat hepatic SULT2A enzyme activity (Fang et al., 2005a). The expression of rodent and human hepatic SULT2A is regulated by the Pregnane X Receptor (PXR) (Liu and Klaassen, 1996; Runge-Morris and Kocarek, 2005; Fang et al., 2005a; 2005b; Duanmu et al., 2002). Dexamethasone administration produced significant increases in rat hepatic SULT2A mRNA and protein contents, relative to control (Fang et al., 2005a). Treatment of human hepatocyte cultures with PXRactivating concentrations of dexamethasone and rifampicin both produced concentration-dependent increases in human SULT2A1 mRNA and protein contents (Fang et al., 2005a; 2005b; Duanmu et al., 2002). In addition, more and more recent data suggest 
Am. J. Pharm. \& Toxicol., 5 (3): 125-132, 2010

that hepatic SULT gene expression is regulated by the same transcription factor networks that also control the expression of the Cytochrome P450s (CYP), such as the Aryl hydrocarbon (Ah) receptor and members of the nuclear receptor superfamily, such as PXR, CAR and PPAR $\alpha$, although the co-regulation between the SULT and CYP gene families doesn't always occur in a coordinate direction (Runge-Morris, 1998; Runge-Morris and Kocarek, 2005; Jain et al., 2008).

Endogenous hormones including glucocorticoids and cytokines are under CNS control. METH is a CNS stimulant which has been shown to produce long-term changes in numerous measures of both dopaminergic and serotonergic function such as enzyme activity, dopamine content and monoamine transmitters (Quinton and Yamamoto, 2006; Gold et al., 2009; Krasnova and Cadet, 2009; Guilarte et al., 2003; Kish, 2008; Kita et al., 2003; Cadet et al., 2007; Volz et al., 2007; Fleckenstein et al., 2007; Sofuoglu and Sewell, 2009). METH increase synaptic levels of the monoamines Dopamine (DA), serotonin and Norepinephrine (NE) (Sofuoglu and Sewell, 2009). It has been demonstrated that dopaminergic system can regulate CYP isoenzymes expression in liver by altering levels of pituitary hormones and cytokine (Wojcikowski, 2004; Wojcikowski et al., 2007; 2008; Konstandi et al., 2008). Psychostimulant amphetamine has been shown to cause a large increase in corticosterone concentration leading to a substantial rise in CYP3A activity and protein level by activating PXR (Wojcikowski et al., 2008). Being one of important endogenous glucocorticoids, corticosterone may regulate SULT1A1 and SULT2A1 by acting on GR and PXR respectively, in the similar way as to dexamethasone. As the analog of amphetamine, METH is also an important member of the family of phenethylamines. METH has similar chemical structure to amphetamine and they have same pharmacology action mechanism. So it is highly possible that METH administration may change the corticosterone concentration in vivo and regulate rSULTs through GR or PXR. Our results shows that rSULT1A1, rSULT2A1 and rSULT1E1 were induced in liver and brain of both male and female rats following METH treatment at low and middle doses treatment for 7 days. At these doses range treatment, the induction of rSULTs by METH may be mediated through the dopamine-related signaling pathway. METH changes the dopamine content in brain and caused increase in corticosterone (and or other hormones) concentration leading to a substantial rise in SULTs expression by activating PXR or GR. It is known that 4-Hydroxymethamphetamine (4-OH-METH) is one of the main metabolites of
METH and 4-OH-METH is the substrate of SULT1A1. The induction of CYP3A and SULTs will enhance the detoxification of METH.

\section{CONCLUSION}

Our data suggest that the central dopaminergic system may play an important role in the regulation of liver and brain rSULTs. This influence of brain dopaminergic system on the rSULTs can have physiological and toxicological significance, since rSULTs are important for drug detoxification and endogenous biosignaling molecules regulation.

\section{ACKNOWLEDGMENT}

This study was supported in part by National Institutes of Health (NIH) grant GM078606 (G.C.), American Cancer Society grant RSG-07-028-01-CNE (G.C.), United States Department of Agriculture (USDA) grant 2006-35200-17137 (G.C.), Oklahoma Center for the Advancement of Science and Technology (OCAST) grant HR05-015 (G.C.) and National Natural Science Foundation of China (NSFC) grant 81072699 (T.Z.).

\section{REFERENCES}

Bojarova, P. and S.J. Williams, 2008. Sulfotransferases, sulfatases and formylglycine-generating enzymes: A sulfation fascination. Curr. Opin. Chem. Biol., 12: 573-581.

http://cat.inist.fr/?aModele $=$ afficheN\&cpsidt $=2082$ 0278

Cadet, J.L., I.N. Krasnova, S. Jayanthi and J. Lyles, 2007. Neurotoxicity of substituted amphetamines: Molecular and cellular mechanisms. Neurotox Res., 11: 183-202. PMID: 17449459

Chapman, E., M.D. Best, S.R. Hanson and C. Wong, 2004. Sulfotransferases: Structure, mechanism, biological activity, inhibition and synthetic utility. Angewandte Chem., Int. Edn., 43: 3526-3548. DOI: 10.1002/anie.200300631

Chen, Y., C. Huang, T. Zhou and G. Chen, 2008. Genistein induction of human sulfotransferases in HepG2 and Caco-2 cells. Basic Clin. Pharm. Toxicol., 103: 553-559. DOI: 10.1111/j.17427843.2008.00316.x

Duanmu, Z., D. Locke, J. Smigelski, W. Wu, M.S. Dahn et al., 2002. Effects of dexamethasone on aryl (SULT1A1)-and hydroxysteroid (SULT2A1)-sulfotransferase gene expression in primary cultured human hepatocytes. Drug Metab. Dispos., 30: 997-1004. DOI: 10.1124/dmd.30.9.997 
Duanmu, Z., T.A. Kocarek and M. Runge-Morris, 2001. Transcriptional regulation of rat hepatic aryl Sulfotransferase (SULT1A1) gene expression by glucocorticoids. Drug Metab. Dispos., 29: 1130-1135. PMID: 11454732

Dunn, R.T. and C.D. Klaassen, 2000. Thyroid hormone modulation of rat sulphotransferase mRNA expression. Xenobiotica, 30: 345-357. DOI: 10.1080/004982500237550

Fang, H.L., M. Abdolalipour, Z. Duanmu, J.R. Smigelski, A. Weckle et al., 2005a. Regulation of glucocorticoid-inducible hydroxysteroid Sulfotransferase (SULT2A-40/41) gene transcription in primary cultured rat hepatocytes: Role of CCAAT/enhancer-binding protein liverenriched transcription factors. Drug Metab. Dispos., 33: 147-156. DOI: 10.1124/dmd.104.000281

Fang, H.L., S.C. Strom, H. Cai, C.N. Falany, T.A. Kocarek et al., 2005b. Regulation of human hepatic hydroxysteroid sulfotransferase gene expression by the peroxisome proliferator-activated receptor alpha transcription factor. Mol. Pharm., 67: 1257-1267. DOI: $10.1124 / \mathrm{mol} .104 .005389$

Fleckenstein A.E., T.J. Volz, E.L. Riddle, J.W. Gibb and G.R. Hanson, 2007. New insights into the mechanism of action of amphetamines. Annu. Rev. Pharmacol. Toxicol., 47: 681-698. DOI: 10.1146/annurev.pharmtox.47.120505.105140

Gallucci, R.M. and B.M. Mickle, 2006. Inflammatory cytokine expression patterns in rat skin exposed to JP-8 jet fuel. Am. J. Pharmacol. Toxicol., 1: 48-53. http://scipub.org/fulltext/AJPT/AJPT1348-53.pdf

Gamage, N., A. Barnett, N. Hempel, R.G. Duggleby, K.F. Windmill et al., 2006. Human sulfotransferases and their role in chemical metabolism. Toxicol. Sci., 90: 5-22. PMID: 16322073

Gold, M.S., F.H. Kobeissy, K.K.W. Wang, L.J. Merlo and A.W. Bruijnzeel et al., 2009. Methamphetamine- and trauma-induced brain injuries: Comparative cellular and molecular neurobiological substrates. Biol. Psychiatry, 66: 118-127. PMID: 19345341

Guilarte, T.R., M.K. Nihei, J.L. McGlothan and A.S. Howard, 2003. Methamphetamine-induced deficits of brain monoaminergic neuronal markers: Distal axotomy or neuronal plasticity. Neuroscience, 122: 499-513. PMID: 14614914

Hempel, N., N. Gamage, J.L. Martin and M.E. McManus, 2007. Human cytosolic sulfotransferase SULT1A1. Int. J. Biochem. Cell Biol., 39: 685-689. PMID: 17110154
Jain, S., G. Khare, P. Tripathi and A.K. Tyagi, 2008. An inducible system for the identification of target genes for a regulator in mycobacteria. Am. J. Biochem. Biotechnol., 4: 226-230. http://www.scipub.org/fulltext/ajbb/ajbb43226-230.pdf

Kauffman, F.C., 2004. Sulfonation in pharmacology and toxicology. Drug Metab. Rev., 36: 823-843. DOI: $10.1081 / \mathrm{DMR}-200033496$

Kish, S.J., 2008. Pharmacologic mechanisms of crystal meth. Can. Med. Assoc. J., 178: 1679-1682. PMID: 18559805

Kita, T., G.C. Wagner and T. Nakashima, 2003. Current research on methamphetamine-induced neurotoxicity: Animal models of monoamine disruption. J. Pharm. Sci., 92: 178-195. DOI: 10.1254/jphs.92.178

Klaassen, C.D., L. Liu and R.T. Dunn, 1998. Regulation of sulfotransferase mRNA expression in male and female rats of various ages. Chem. Biol. Interact., 109: 299-313. PMID: 9566754

Konstandi, M., P. Harkitis, D. Kostakis, M. Marselos and E.O. Johnson et al., 2008. D2-receptor-linked signaling pathways regulate the expression of hepatic CYP2E1. Life Sci., 82: 1-10. PMID: 17988693

Krasnova, I.N. and J.L. Cadet, 2009. Methamphetamine toxicity and messengers of death. Brain Res. Rev., 60: 379-407. PMID: 19328213

Liu, L. and C.D. Klaassen, 1996. Regulation of hepatic sulfotransferases by steroidal chemicals in rats. Drug Metab. Dispos., 24: 854-858. PMID: 8869819

Lu, J.H., H.T. Li, M.C. Liu, J.P. Zhang and M. Li et al., 2005. Crystal structure of human sulfotransferase SULT1A3 in complex with dopamine and 3'phosphoadenosine 5'-phosphate. Biochem. Biophys. Res. Commun., 335: 417-423. PMID: 16083857

Maiti, S. and G. Chen, 2003a. Methotrexate is a novel inducer of rat liver and intestinal sulfotransferases. Arch. Biochem. Biophys. 418: 161-168. PMID: 14522587

Maiti, S. and G. Chen, 2003b. Tamoxifen induction of aryl sulfotransferase and hydroxysteroid sulfotransferase in male and female rat liver and intestine. Drug Metab. Dispos., 31: 637-644. DOI: 10.1124/dmd.31.5.637

Fisher, M.A., B.B. Plikaytis and T.M. Shinnick, 2005. Evaluation of transcript labeling techniques and development of a membrane-based parallel gene expression assay. Am. J. Biochem. Biotechnol., $\quad$ 1: 5-12. http://www.scipub.org/fulltext/ajbb/ajbb115-12.pdf 
Niculescu, A.B., D.S. Segal, R. Kuczenski, T. Barrett and R.L. Hauger et al., 2000. Identifying a series of candidate genes for mania and psychosis: A convergent functional genomics approach. Physiol. Genom., 4: 83-91. PMID: 11074017

Quinton, M.S. and B.K. Yamamoto, 2006. Causes and consequences of methamphetamine and MDMA toxicity. AAPS J., 8: E337-E347. DOI: 10.1208/aapsj080238

Rath, V.L., D. Verdugo and S. Hemmerich, 2004. Sulfotransferase structural biology and inhibitor discovery. Drug Discov. Today, 9: 1003-1011. DOI: 10.1016/S1359-6446(04)03273-8

Runge-Morris, M. and T.A. Kocarek, 2005. Regulation of sulfotransferases by xenobiotic receptors. Curr. Drug Metab., 6: 299-307. DOI: 10.2174/1389200054633871

Runge-Morris, M., 1998. Regulation of sulfotransferase gene expression by glucocorticoid hormones and xenobiotics in primary rat hepatocyte culture. Chem.-Biol. Interact., 109: 315-327. DOI: 10.1016/S0009-2797(97)00142-7

Runge-Morris, M., W. Wu and T.A. Kocarek, 1999. Regulation of rat hepatic hydroxysteroid Sulfotransferase (SULT2-40/41) gene expression by glucocorticoids: Evidence for a dual mechanism of transcriptional control. Molecular Pharmacol., $\quad$ 56: 1198-1206. http://molpharm.aspetjournals.org/content/56/6/119 8.short

Salman, E.D., S.A. Kadlubar and C.N. Falany, 2009. Expression and localization of cytosolic Sulfotransferase (SULT) 1A1 and SULT1A3 in normal human brain. Drug Metab. Dispos., 37: 706-709. DOI: 10.1124/dmd.108.025767

Santini, F., D. Cortelazzi, A.M. Baggiani, A.M. Marconi and P. Beck-Peccoz et al., 1993. A study of the serum 3,5,3'-triiodothyronine sulfate concentration in normal and hypothyroid fetuses at various gestational stages. J. Clin. Endocrinol. Metab., 76: $1583-1587$.

http://cat.inist.fr/?aModele $=$ afficheN\&cpsidt $=4808$ 079

Sofuoglu, M. and R.A. Sewell, 2009. Norepinephrine and stimulant addiction. Addict. Biol., 14: 119-129. DOI: $10.1111 / \mathrm{j} .1369-1600.2008 .00138 . x$

Tolwani, R.J., M.W. Jakowec, G.M. Petzinger, S. Green and K. Waggie, 1999. Experimental models of Parkinson's disease: Insights from many models. Lab. Anim. Sci., 49: 363-371. http://cat.inist.fr/?aModele $=$ afficheN\&cpsidt=1019 6871
Volz, T.J., A.E. Fleckenstein and G.R. Hanson, 2007. Methamphetamine-induced alterations in monoamine transport: Implications for neurotoxicity, neuroprotection and treatment. Addiction, $\quad 102$ : 44-48. http://onlinelibrary.wiley.com/doi/10.1111/j.13600443.2007.01771.x/pdf

Wang, L.Q. and M.O. James, 2006. Inhibition of sulfotransferases by xenobiotics. Curr. Drug Metab., 7: 83-104. PMID: 16454694

Wojcikowski, J., 2004. Potential role of the brain dopaminergic system in the regulation of cytochrome P-450 expression. Polish J. Pharm., 56: 701-708.

http://cat.inist.fr/?aModele $=$ afficheN\&cpsidt $=1654$ 3362

Wojcikowski, J., K. Gołembiowska and W. Daniel, 2007. The regulation of liver cytochrome p450 by the brain dopaminergic system. Curr. Drug Metab., 8: 631-638. DOI: $10.2174 / 138920007781368872$

Wojcikowski, J., K. Gołembiowska and W. Daniel, 2008. Regulation of liver cytochrome P450 by activation of brain dopaminergic system: Physiological and pharmacological implications. Biochem. Pharm., 76: 258-267. http://cat.inist.fr $/$ ?aModele $=$ afficheN\&cpsidt $=2050$ 2232

Wu, W., T.A. Kocarek and M. Runge-Morris, 2001. Sex-dependent regulation by dexamethasone of murine hydroxysteroid sulfotransferase gene expression. Toxicol. Lett., 119: 235-246. http://cat.inist.fr $/$ ?aModele $=$ afficheN\&cpsidt $=9233$ 59

Wu, S.Y., D.H. Polk, W.S. Huang, E. Ho and J.M. Kattan et al., 2008. 3'-monoiodothyronine sulfate and Triac sulfate are thyroid hormone metabolites in developing sheep. Pediatr. Res., 63: 149-153. http://cat.inist.fr/?aModele $=$ afficheN\&cpsidt $=2001$ 7076

Yanai, H., H. Yoshida, H. Fujiwara, S. Yoshida and H. Fuda et al., 2005. Oxidized low-density lipoprotein upregulates GM2 activator protein gene expression. Am. J. Biochem. Biotechnol., 1: 90-94. http://www.scipub.org/fulltext/ajbb/ajbb1290-94.pdf

Yasuda, S., T. Yasuda, Y. Hui, M.Y. Liu and M. Suiko et al., 2009. Concerted action of the cytosolic sulfotransferase, SULT1A3 and catechol-Omethyltransferase in the metabolism of dopamine in SK-N-MC human neuroblastoma cells. Neurosci. Res., 64: 273-279. http://cat.inist.fr/?aModele $=$ afficheN\&cpsidt=2155 9182 